\title{
Horizontal Hippocampal Slices of the Mouse Brain
}

\author{
Evelien Van Hoeymissen ${ }^{1,2}$, Koenraad Philippaert ${ }^{2}$, Rudi Vennekens ${ }^{2}$, Joris Vriens ${ }^{*}, 1$, Katharina Held ${ }^{*}, 1,2$ \\ ${ }^{1}$ Laboratory of Endometrium, Endometriosis and Reproductive Medicine, Department of Development and Regeneration, KU Leuven ${ }^{2}$ Laboratory of lon \\ Channel Research, VIB-KU Leuven Center for Brain and Disease Research, Leuven, Belgium and Department of Molecular Medicine, KU Leuven \\ *These authors contributed equally
}

\section{Corresponding Author}

Katharina Held

kathi.held@kuleuven.vib.be

\section{Citation}

Van Hoeymissen, E., Philippaert, K., Vennekens, R., Vriens, J., Held, K. Horizontal Hippocampal Slices of the Mouse Brain. J. Vis. Exp. (163), e61753, doi:10.3791/61753 (2020).

\section{Date Published}

September 22, 2020

DOI

$10.3791 / 61753$

URL

jove.com/video/61753

\section{Abstract}

The hippocampus is a highly organized structure in the brain that is a part of the limbic system and is involved in memory formation and consolidation as well as the manifestation of severe brain disorders, including Alzheimer's disease and epilepsy. The hippocampus receives a high degree of intra- and inter-connectivity, securing a proper communication with internal and external brain structures. This connectivity is accomplished via different informational flows in the form of fiber pathways. Brain slices are a frequently used methodology when exploring neurophysiological functions of the hippocampus. Hippocampal brain slices can be used for several different applications, including electrophysiological recordings, light microscopic measurements as well as several molecular biological and histochemical techniques. Therefore, brain slices represent an ideal model system to assess protein functions, to investigate pathophysiological processes involved in neurological disorders as well as for drug discovery purposes.

There exist several different ways of slice preparations. Brain slice preparations with a vibratome allow a better preservation of the tissue structure and guarantee a sufficient oxygen supply during slicing, which present advantages over the traditional use of a tissue chopper. Moreover, different cutting planes can be applied for vibratome brain slice preparations. Here, a detailed protocol for a successful preparation of vibratomecut horizontal hippocampal slices of mouse brains is provided. In contrast to other slice preparations, horizontal slicing allows to keep the fibers of the hippocampal input path (perforant path) in a fully intact state within a slice, which facilitates the investigation of entorhinal-hippocampal interactions. Here, we provide a thorough protocol for the dissection, extraction, and acute horizontal slicing of the murine brain, and discuss challenges and potential pitfalls of this technique. Finally, we will show some examples for the use of brain slices in further applications. 


\section{Introduction}

The extensive exploration of the hippocampus started when Scoville and Milner reported the inability of a patient (H.M.) to form new, declarative memory after surgical removal of the hippocampus and nearby temporal lobe structures as a treatment for severe epilepsy ${ }^{1}$. From that moment on, the hippocampus has been studied extensively starting from general neuronal properties and functions up to the development of severe brain disorders, such as epilepsy and Alzheimer's disease ${ }^{2,3,4,5}$. The hippocampus is part of the limbic system, consisting of a group of related brain structures involved in emotion and memory formation ${ }^{6,7}$.
A dense network of several fiber pathways accomplishes a tight hippocampal connectivity to internal and external brain structures. These pathways include the medial and lateral perforant path (entorhinal cortex to dentate gyrus, CA3 CA1 and subiculum) ${ }^{8}$, the mossy fiber path (dentate gyrus to CA3) ${ }^{9}$ and the Schaffer collateral/associational commissural pathway (CA3 to $\mathrm{CA} 1)^{10}$ (Figure 1). The hippocampus presents one of the most broadly explored brain areas so far because of its highly conserved laminar organization of the neuronal layer formation, and the possibility to obtain vital neuronal cultures and brain slices with relative ease ${ }^{5}$.

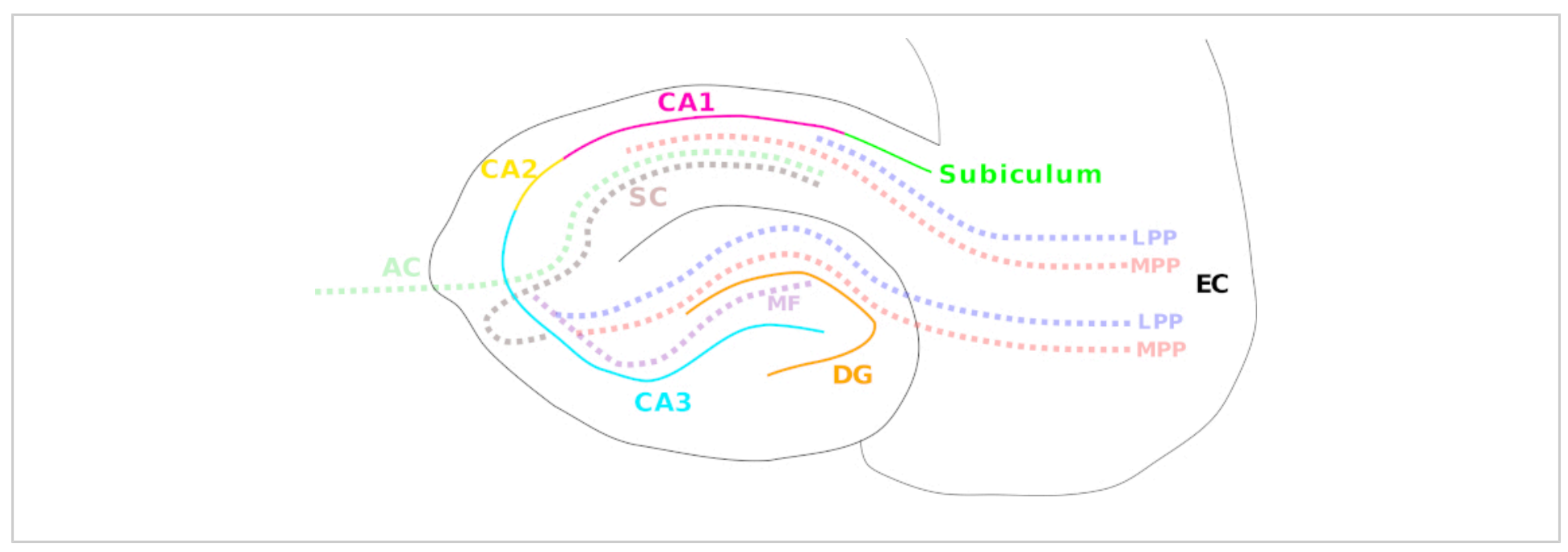

Figure 1: Cartoon illustrating the different hippocampal regions and main fiber pathways. The different hippocampal regions are indicated by solid colored lines: entorhinal cortex (EC; black), dentate gyrus (DG; orange), Cornu Ammonis (CA) 3 (cyan), 2 (yellow), and 1 (magenta), and the subiculum (green). Fiber pathways are shown with a colored dotted line: the medial (MPP, red) and lateral perforant path (LPP, blue) (from the entorhinal cortex to the dentate gyrus, CA3, CA1, and subiculum), the mossy fiber pathway (MF, violet) (from the dentate gyrus to CA3) and the Schaffer collateral (SC, brown) (ipsilateral from CA3 to CA1)/associational commissural pathways (AC, light green) (contralateral from CA3 to CA1). Please click here to view a larger version of this figure.

Brain slice protocols often result in the loss of connections from more distant brain areas to the area of interest ${ }^{5}$.
Moreover, the capillaries are no longer functional ${ }^{5}$ and the blood circulation is deprived ${ }^{11}$. Despite these limitations, 
brain slices are still primarily used for the investigation of neurophysiological functions of the hippocampus due to a number of advantages. First, the extraction of the hippocampus is fast ${ }^{12}$ and does not require many materials. The only essential instruments include a dissection kit, a laboratory water bath, access to carbogen and a vibrating microtome (vibratome) ${ }^{13}$. Other assets of the brain slice technique are the circumvention of the bloodbrain-barrier (BBB) and the wash-out of endogenously released molecules before the start of the experiment ${ }^{5}$, which makes it possible to study the effect of drugs with relatively precise dosage control ${ }^{14}$. Furthermore, brain slices preserve the cyto-architecture and synaptic circuits within the hippocampus ${ }^{15}, 16$, where the neuroanatomy and the local environment with neuronal connectivity and complex neuron-glia interactions are preserved $4,11,17$. Additionally, hippocampal fiber connections are predominantly unidirectional and hippocampal neurons have a high synaptic plasticity, which tremendously simplifies the collection and interpretation of high-quality electrophysiological recordings in order to understand neurological processes ${ }^{18,19}$. Importantly, brain slices present a valuable asset applicable in a wide range of different scientific techniques, spanning from molecular biological techniques over imaging recordings up to electrophysiological measurements $12,20,21,22,23,24,25,26$.

As outlined above, hippocampal brain slices present a powerful experimental tool to study structural and functional features of the synaptic connectivity. This offers the opportunity to assess the effects of chemicals or mutations on neuronal excitability and plasticity ${ }^{16}$.

Acute brain slice preparations are presenting a relatively sensitive technique and optimal slice quality is highly dependent on ideal experimental conditions, including the age of the animal, the method of euthanasia, the speed of dissection and slicing, the slicing solutions and parameters (e.g., slicing speed) as well as the conditions for slice recovery ${ }^{4}$. Therefore, a well-designed protocol is of uttermost importance and secures the reproducibility across different research units ${ }^{13}$.

Here, we provide a detailed protocol for acute horizontal hippocampal slice preparations, with the aim to preserve the integrity of the hippocampal lateral and medial perforant path and the mossy fiber pathway, allowing the investigation of dentate gyrus related processes ${ }^{9}$. We will describe in detail the key steps to dissect, extract, and horizontally slice the murine brain, followed by representative results of calciummicrofluorimetric recordings and field excitatory postsynaptic potential recordings (fEPSPs) under baseline conditions and during LTP induction protocols in brain slices of wild type C57BL/6J mice.

\section{Protocol}

All animal experiments for this study were approved by the ethical review committee of the $\mathrm{KU}$ Leuven (Belgium) (P021/2012).

\section{Preparation of high-sucrose slice solution and artificial cerebrospinal fluid (ACSF)}

1. Prior to experimental day

1. Prepare $1 \mathrm{~L}$ of $10 \mathrm{x}$ slice pre-solution with laboratory grade Type 1 water containing (in $\mathrm{mM}$ ): $25 \mathrm{KCl}, 20$ $\mathrm{CaCl}_{2}, 10 \mathrm{MgSO}_{4}, 12.5 \mathrm{KH}_{2} \mathrm{PO}_{4}$ (Table 1). In order to prevent calcium phosphate precipitation, slowly mix the chemicals in a beaker pre-filled with $800 \mathrm{~mL} \mathrm{H}_{2} \mathrm{O}$ 
while constantly stirring with a magnetic stirrer. Store the solution at $4{ }^{\circ} \mathrm{C}$ or room temperature (RT).

2. On the experimental day

1. Prepare $1 \mathrm{~L}$ of $1 \mathrm{x}$ ACSF with laboratory grade Type 1 water containing (in $\mathrm{mM}$ ): $125 \mathrm{NaCl}, 2.5 \mathrm{KCl}, 2$ $\mathrm{CaCl}_{2}, 1 \mathrm{MgSO}_{4}, 1.25 \mathrm{NaH}_{2} \mathrm{PO}_{4}, 26 \mathrm{NaHCO}_{3}, 25$ Glucose (Table 2). Use a vapor pressure osmometer to validate the osmolarity between $305-315$ mOsm ( $\mathrm{pH} \sim 7.55-7.6)$.

NOTE: In order to prevent calcium phosphate precipitation, slowly mix all solid chemicals in a beaker pre-filled with $800 \mathrm{~mL}$ of $\mathrm{H}_{2} \mathrm{O}$ while constantly stirring with a magnetic stirrer. Add $\mathrm{MgSO}_{4}$ and $\mathrm{CaCl}_{2}$ at the very end, slowly dripping in the necessary amount from $1 \mathrm{M}$ stock solutions.

2. Continuously bubble $1 \mathrm{x}$ ACSF solution at RT with carbogen to set $\mathrm{pH}$ between 7.3-7.4.

NOTE: If the $\mathrm{pH}$ is slightly too high or too low, small adjustments in the carbogenation strength would be sufficient. If the $\mathrm{pH}$ is higher than 7.45 with carbogenation, adjust it by adding few drops of $1 \mathrm{M}$ $\mathrm{NaH}_{2} \mathrm{PO}_{4}$ solution.

3. Prepare $250 \mathrm{~mL}$ (per brain) of $1 \mathrm{x}$ high-sucrose slice solution in a beaker containing $25 \mathrm{~mL}$ of $10 x$ slice pre-solution and (in mM): 252 Sucrose, $26 \mathrm{NaHCO}_{3}$, and 10 Glucose (Table 3). Verify that the osmolarity is between $320-325 \mathrm{mOsm}(\mathrm{pH} \sim 7.55-7.6)$.

4. Bubble the high-sucrose slice solution for $10-15 \mathrm{~min}$ with carbogen to control the $\mathrm{pH}$ between 7.3-7.4.

NOTE: If the $\mathrm{pH}$ is higher than 7.45 with carbogenation, adjust it by adding a few drops of $1 \mathrm{M}$ $\mathrm{KH}_{2} \mathrm{PO}_{4}$ solution.
5. Store the high-sucrose slice solution for 20-30 $\mathrm{min}$ in the ultra freezer $\left(-80^{\circ} \mathrm{C}\right)$ until it is partially frozen.

\section{Preparation of the workspace for the brain dissection}

1. During the cool-down of the high sucrose slice solution, prepare the following.

1. Warm up the water bath to $32^{\circ} \mathrm{C}$.

2. Fill the recovery chamber (Figure 2A) with the carbogenated ACSF solution and place the chamber in the water bath. Continuously apply carbogen to the main ACSF bottle and the ACSF in the recovery chamber.

3. Usher the animal to the experimental room.

NOTE: The age, sex, and strain of the animal have to be determined by the individual experimenter and is dependent on the specific study question. However, the parameters of the animal should stay constant within one study in order to guarantee comparability between the different experimental days. This protocol was designed for the use of C57BL/6J male mice at the age of 2-6 weeks. If older animals will be used, slice and recovery solutions may have to be adapted accordingly ${ }^{4}, 27$ (e.g., NMDG ${ }^{+}$based solutions ${ }^{23}, 28$ ) in order to preserve the brain health of the acute slices.

4. Prepare the anesthesia chamber.

5. Lay out tissue paper, plastic Pasteur pipette with wide opening (cut open), $90 \mathrm{~mm}$ culture dish filled with ice, a square of filter paper on top of the chilled culture dish, strong scissors for decapitation, dissection scissors, curved forceps, spatula, $35 \mathrm{~mm}$ culture dish, fine brush, blade, specimen plate (comes 
with vibratome), super glue, pipette tip, four straps of filter paper $(\sim 2 \mathrm{~cm} \times 0.5 \mathrm{~cm})$ (Figure 2A).

6. Set up the vibratome: First, program the vibratome for the correct settings (blade travel speed: $0.08 \mathrm{~mm} / \mathrm{s}$, cutting amplitude: $1.4 \mathrm{~mm}$, cutting frequency: $85 \mathrm{~Hz}$ ) and attach the carbogen line in the slice chamber. Next, place the slice chamber in the holder, fill the holder surrounding the slice chamber with ice and attach everything to the vibratome. Finally, place the razor blade in the vibratome blade holder (Figure 2B).

2. After freezing of the high-sucrose solution perform the following steps.

1. After $20-30 \mathrm{~min}$, take the high-sucrose slice solution out of the $-80^{\circ} \mathrm{C}$ ultra-freezer and keep the beaker on ice.

2. Place the high-sucrose solution next to the vibratome on your bench, crush and mix the partially frozen solution with a spatula to get a nice slush and start bubbling with carbogen.

3. Take up some high-sucrose slice solution with the plastic Pasteur pipette to soak the squared filter paper on top of the chilled $90 \mathrm{~mm}$ culture dish.

4. Fill the $35 \mathrm{~mm}$ culture dish (or any equivalent small container that is suitable to store the whole brain) up to $75 \%$ with the high-sucrose slice solution (sufficient to cover the whole brain) and cool the culture dish on ice kept next to the beaker with the rest of the solution. Carbogenate the solution in the $35 \mathrm{~mm}$ culture dish.

\section{Dissection and positioning of the murine brain}

1. Anesthetize the animal with $5 \%$ isoflurane. Determine the proper depth of anesthesia by pinching the paw. No paw withdrawal reflex should occur.
2. Transfer the animal on tissue paper and decapitate it with strong scissors or a small animal guillotine.

3. Use dissection scissors to cut open the scalp.

4. Cut open the calvaria along the sagittal suture and remove it with the help of curved forceps until the whole brain, including the olfactory bulbs, is visible.

NOTE: Be careful to not damage the brain with the sharp edges of the calvaria or forceps.

5. Use a spatula to carefully scoop out the brain (olfactory bulbs should stay attached).

6. Transfer the brain in the chilled $35 \mathrm{~mm}$ culture dish and remove all hair or blood particles from the tissue by gently washing the brain with an ACSF-filled Pasteur pipette (blood has cytotoxic effects on brain tissue ${ }^{29}$ ).

7. Transfer the brain with the help of the spatula on the soaked filter paper on top of the chilled $90 \mathrm{~mm}$ culture dish (Figure 2A).

8. Use a blade to cut the brain in half, separating the two hemispheres, and place both hemispheres on the freshly cut medial side.

9. Use a blade to remove the dorsal part $(5 \%-10 \%)$ of the brain from each hemisphere with a parallel cut to the dorsal top (Figure 2C) ${ }^{30}$ and place both hemispheres on the freshly cut side with the ventral part of the brain facing upwards.

10. Position a drop of super glue on the specimen plate and spread out properly with a pipette tip to accommodate both the hemispheres.

11. Use a filter paper strap to pick up one hemisphere with capillary forces by touching the ventral side with the filter paper strap, thereby not damaging the tissue. 
12. Use another filter paper strap to carefully semi-dry the dorsal side of the brain before positioning the hemisphere with the dorsal side down on top of the glue on the specimen plate. Repeat the same procedure with the second hemisphere.

NOTE: The hemispheres should be positioned in horizontal alignment in a mirrored fashion on the vibratome plate, with the rostral sides pointing toward the outside and the caudal sides facing (but not touching) each other in the middle. The medial sides of both hemispheres should point toward the vibratome blade and the lateral sides toward the experimenter (Figure 2D).

13. Place the specimen plate in the slicing chamber and quickly, but carefully, cover it with ice-cold high-sucrose slice solution slush. As soon as the solution touches the glue, it will solidify and properly glue the hemispheres to the specimen plate.

14. Assure that the hemispheres are properly covered with high-sucrose slice solution and confirm that the solution is bubbled with carbogen.

NOTE: The entire dissection procedure should be performed as fast as possible. Please ensure that the brain does not stay without the supply of oxygen for a very long time. It should take only around 1-1.5 min from decapitation to brain submersion in the high-sucrose slice solution slush. This is the most critical requirement for acute brain slice preparations in order to warrant high quality of the slice.

\section{Horizontal slicing of the brain}

1. Position the vibratome blade in front of the medial side of the hemispheres and lower it to the same height as the ventral sides of the hemispheres that are now facing upwards. Lower the blade with the help of the vibratome control to $600 \mu \mathrm{m}$ further in the dorsal direction and start slicing. The blade should hit the tissue (if not, reverse the blade and lower it a bit more). Slice until the first two slices are completely separated from the two hemispheres.

2. Reverse the blade and lower another $300 \mu \mathrm{m}$ and slice again.

3. When the hippocampus becomes visible (use the mouse $^{31}$ brain atlas for help, if necessary) (Figure 2E) collect the slices with the widened plastic Pasteur pipette. Collect the slices until the caudate putamen becomes visible next to the hippocampus. Typically, between 8-12 slices of $300 \mu \mathrm{m}$ (4-6 per hemisphere) can be collected for the mouse brain.

4. Use the plastic Pasteur pipette to collect the slices and transfer them to the recovery chamber in the water bath (Figure 2F) (collect slices after each round of slicing to prevent them from floating around in the slice chamber). NOTE: Work as fast as possible and ensure that the high-sucrose slice solution is ice-cold and carbogenated during the entire procedure. If necessary, refill the ice surrounding the slice chamber.

\section{Recovery of brain slices for electrophysiological recordings}

1. Leave the slices in the ACSF-filled recovery chamber in the $32{ }^{\circ} \mathrm{C}$ water bath for $1 \mathrm{~h}$.

NOTE: Recovery chambers are also commercially available.

2. Take the recovery chamber out of the water bath.

3. Place the slices at RT for at least another $30 \mathrm{~min}$ for recovery before starting any further application. 


\section{6. fEPSP recordings in the medial perforant path (MPP) of the hippocampus}

1. Pull recording pipettes from borosilicate glass capillaries with a horizontal pipette puller to receive pipettes of the size of $\sim 2 \mathrm{M} \Omega$ when filled with ACSF solution and immersed in the ACSF-filled recording chamber.

2. Fill ACSF bath solution and ACSF solution containing the appropriate chemicals (e.g., Bicuculline) in a gravitycontrolled multi-barrel perfusion system that is connected to the recording chamber. Continuously carbogenate all the solutions.

3. Turn on a peristaltic or vacuum pump that is connected to a suction tubing that ends opposite of the perfusion line in the recording chamber. Open the ACSF-filled barrel and start to establish a continuous flow (1-2 drops per second). Verify that the reference electrode is submerged in the ACSF solution.

4. Switch on the setup computer, the amplifier, the micromanipulator, the stimulator, the microscope light(s), the camera, and the monitor (if applicable).

5. Open the appropriate software for electrophysiological recordings (several different hardware and software providers for electrophysiological equipment exist).

6. Transfer one brain slice hemisphere from the recovery chamber to the recording chamber of the slice setup and position it in the correct orientation with the dentate gyrus granule cell layer and the molecular layer in the field of view. Make sure that the stimulation electrode can reach the MPP in the slice from the direction of the entorhinal cortex and that the recording electrode has access to the MPP from the exact opposite side from the direction of CA3.
7. Stabilize the slice in the recording chamber with a paper clip (Figure $\mathbf{3 A}$ ) or a commercially available brain slice grid.

NOTE: It can be helpful to turn off the perfusion during slice transfer and positioning. However, this should not take too long in order to guarantee the quality of the brain slice.

8. Lower and position the recording and stimulation electrodes in the MPP in the lower third of the molecular layer close to the granule cell layer (Figure 3B), facing each other at a distance of approximately $100-150 \mu \mathrm{m}$. The stimulation electrode should minimally contact the surface, while the recording electrode should slightly infiltrate the upper slice layer.

9. Apply a low intensity stimulus $(30-50 \mu \mathrm{A}$ for $0.1 \mathrm{~ms})$ to the brain slice in order to obtain the fEPSP signal. Adapt the position of the electrodes if necessary (e.g., low or atypical shaped fEPSP signal).

10. Start recording Input-Output curves: apply increasing stimulus intensities to the brain slice in intervals of $30 \mathrm{~s}$.

11. Set the stimulus intensity to $50 \%$ of the maximal fEPSP response and start baseline fEPSP recordings applying the $50 \%$ stimulus intensity for $20-40$ min in 30 s intervals to the brain slice.

12. If the baseline appears to be stable, proceed with additional recordings (e.g., LTP/LTD protocols and/or drug applications). (For LTP induction in the MPP, here a protocol consisting of four times $1 \mathrm{~s} 100 \mathrm{~Hz}$ pulses applied in an interval of 5 min was used. Bicuculline was applied 10 min before and during the conditioning phase.)

NOTE: All recordings should be performed in the current clamp mode with appropriate low-pass filtering $(<5 \mathrm{kHz})$ and sampling rates $(>10 \mathrm{kHz})$. 
13. Extract the data in an appropriate file format and analyze fEPSP parameters, such as fiber volley, fEPSP amplitude, and fEPSP slope.

\section{Calcium imaging recordings of brain slices}

1. Transfer one brain slice hemisphere in a 12-well chamber and load it for $30 \mathrm{~min}$ to $1 \mathrm{~h}$ with an appropriate calcium dye. Continuously carbogenate the loading solution by inserting a carbogenation tube through a self-made hole (with a hot $18 \mathrm{G}$ needle) in the lid of the 12-well plate.

NOTE: This step is not necessary in case of brain slice preparations from genetically modified animals that endogenously express a fluorescent reporter such as GCaMP.

2. Prepare the recording setup as described in steps 6.26.5 .

NOTE: No amplifier or micromanipulator are necessary for microfluorimetric imaging experiments. The use of a stimulator is experiment dependent. Specific software for fluorescence imaging experiments is essential.

3. Adjust the software to the correct imaging settings: this includes camera amplifier and binning setting, setting of the wavelength, exposure time, and timing protocol. Settings can vary depending on the exact experiments. (For the example traces, $1 \times 1$ binning, 2.4x pre-amplifier gain, $300 \mathrm{EM}$ camera gain, exposure of $50 \mathrm{~ms}$ at $488 \mathrm{~nm}$ repeated every $500 \mathrm{~ms}$ for the duration of the experiment was used.)

4. After loading with the calcium dye, transfer the brain slice hemisphere from the 12-well to the recording chamber and position it on the microscope stage.
5. Stabilize the slice in the recording chamber with a paper clip (Figure $\mathbf{3 A}$ ) or a commercially available brain slice grid similar to the one described in step 6.7.

6. Assure that the area of interest is in the correct microscopic field and in focus.

7. Use the fluorescence imaging software to select several regions of interest (ROIs) on your slice.

NOTE: It can be useful to select the whole field of view to follow the general fluctuations in brightness, due to photobleaching.

8. Start the recording and apply the experimental test-drugs at chosen time points.

NOTE: It is advisable to apply a high $\mathrm{K}^{+}$solution at the end of each experiment to verify the neuronal character of the cells and the slice quality.

9. Extract the data in an appropriate file format and analyze the fluorescence signal changes that occur in the ROls over the entire measurement. ROls can also be adapted during the off-line analysis.

NOTE: Other protocols that describe fEPSP recordings and calcium microfluorimetry in brain slices are available in literature $24,32,33,34,35$.

\section{Representative Results}

General overview of tools and critical steps needed for the protocol

Figure 2 presents all the necessary tools and critical steps for the preparation of horizontal acute hippocampal brain slices as described in this protocol. Generally, a limited number of key instruments are required, including a few dissection tools and a slice recovery chamber (Figure 2A), a laboratory water bath, and a vibratome (Figure 2B). Figure 2C-E visualize important steps and orientations of the brain and 
hemispheres during the slice preparation protocol. Figure $2 \mathrm{~F}$ is an illustration of an expected result of horizontal brain slices.

\section{fEPSP recordings in the medial perforant path}

After the recovery period, the brain slices can be used for electrophysiological recordings of fEPSPs. Here, we used an upright microscope equipped with a multi-channel gravity-controlled perfusion system (Figure 3A and Figure 3B). A glass micropipette $(\sim 2 \mathrm{M} \Omega)$ was filled with ACSF solution and attached on top of a chloride-coated silver electrode that is mounted to an operational amplifier in circuit with a chlorinated bath electrode. fEPSPs were recorded and visualized with an amplifier and appropriate recording software by inserting the glass micropipette into the MPP of the hippocampus in the upper layer of the brain slice. fEPSPs were induced by stimulation with a 2-contact cluster microelectrode, applying different current intensities to the MPP upstream of the recording electrode. Note that this protocol is not intended to explain how to obtain MPP recordings, but simply uses recordings in the MPP as an example to demonstrate the success of the slice preparation protocol described here. If someone attempts to perform MPP recordings, certain controls (e.g., paired pulse recordings) might be necessary in order to ensure the proper recording site and distinguish the MPP from the LPP $8,36,37$.

Figure 3C illustrates a negative (left panel, low quality slice) and positive (right panel, high quality slice) example of an fEPSP recording. The negative example trace shows a large fiber volley (FV) amplitude that is even higher than the actual fEPSP amplitude $(\approx 0.5 \mathrm{mV})$. In contrast, the high-quality slice example (right panel) shows a small FV to fEPSP ratio and a high fEPSP amplitude $(>0.5 \mathrm{mV})$. The fiber volley is the signal that occurs upon depolarization of the stimulated neuronal fibers and therefore precedes the postsynaptic potentiation (fEPSP). The relation of FV to fEPSP properties provides important information about the preservation of the axonal and synaptic properties. High quality slices with intact nerve fibers should show a high fEPSP amplitude to FV ratio. On the contrary, low quality slices with impaired conduction properties will have a decreased fEPSP to FV ratio. Similarly, the viability of a brain slice can be analyzed by plotting fEPSP slopes versus the fiber volley amplitudes (Figure 3D).

Moreover, Input-Output curves (fEPSP slope and FV amplitude over stimulus intensity) are standardly used in order to determine the slice quality. Such curves are obtained by applying increasing current stimuli to the brain slice and by monitoring the subsequent fEPSP responses. Low quality brain slices show a reduced Input-Output curve due to suboptimal conduction properties of poorly preserved brain tissue (Figure 3E,F). Furthermore, Input-Output curves are necessary to define the ideal stimulation intensity range for the investigation of synaptic processes. Ideally, the stimulus intensity should be set around $50 \%$ of the intensity for maximal responses. At this chosen stimulus intensity, the fEPSP responses are highly sensitive for any changes in synaptic plasticity, which offers the opportunity to investigate both long-term potentiation (LTP) and long-term depression (LTD).

In order to study synaptic plasticity, the synaptic transmission of the brain slice (fEPSP slope) at the chosen $50 \%$ stimulus intensity is monitored for a longer time period (usually between $20-40 \mathrm{~min}$ ) before the conditioning phase. Viable brain slices will have stable baselines, while brain slices with an unstable baseline cannot be used for further conditioning protocols in order to study synaptic plasticity of the brain circuits (Figure 3G, upper panel). fEPSP baseline recordings 
can also be useful in order to monitor drug effects on synaptic transmission itself (Figure 3G, lower panel). The mean of the recorded fEPSP baseline signals is typically used to normalize an fEPSP time course and is standardly set at $100 \%$

Synaptic plasticity can be studied by applying specific conditioning protocols to the brain slices. These protocols depend on the investigated brain circuit and the mechanism of interest (e.g., LTP or LTD). In order to induce LTP in the MPP of the dentate gyrus, a strong conditioning protocol is necessary due to the strong GABAergic inhibition that is present at the MPP synapses ${ }^{38}$. It is reported that the GABAergic inhibition is even more pronounced in brain slices prepared with high-sucrose slicing solutions ${ }^{39}$. Here, we use a protocol consisting of four stimulations of $1 \mathrm{~s}$ long 100 $\mathrm{Hz}$ pulses applied in a 5 min interval while being treated with the GABAA receptor antagonist Bicuculline (Figure $3 \mathrm{H})$. The co-addition of NMDA and Bicuculline during the conditioning period results in an increased LTP (Figure $3 \mathbf{H}$ ). Low quality of the slice and unstable synaptic transmission (fEPSP baseline) could result in altered or unsuccessful LTP and LTD induction. Therefore, it is of high importance to work with high quality slice preparations and use rigorous exclusion criteria (low fEPSP amplitude to fiber volley ratio
(<3), small fEPSP slope $(<0.5 \mathrm{mV} / \mathrm{ms})$ or amplitude $(<0.5 \mathrm{mV})$ and unstable fEPSP baseline (change of more than $5 \%$ ) for unviable slices when investigating synaptic processes.

\section{Calcium microfluorimetric measurements in the granule cell layer of the dentate gyrus}

After recovery, a brain slice was incubated at room temperature with $2 \mu \mathrm{M}$ of a calcium-sensitive dye for $1 \mathrm{~h}$ in carbogenated ASCF, shielded from light. The slice was transferred into a recording chamber (Figure 3A) on an upright fluorescence microscope equipped with a multichannel gravity-controlled perfusion system. Fluorescence emission images were acquired every 500 milliseconds after illumination at $488 \mathrm{~nm}$ (Figure 4A,B). Excitation was done with a Xenon lamp and a scanner mounted diffraction grating monochromator and image acquisition was performed with a computer-controlled CCD camera. During the measurements, the slice was treated with the NMDA receptor antagonist APV, which resulted in a decrease in the intracellular calcium concentration. Stimulation of the slice with an extracellular solution containing a high potassium concentration $(50 \mathrm{mM})$ resulted in a massive influx of extracellular calcium due to the depolarization of the neurons and the opening of voltagegated ion channels (Figure 4C).

\begin{tabular}{|c|c|c|c|}
\hline Compound & Concentration $(\mathbf{m M})$ & Molecular weight (g/mol) & Amount (g) \\
\hline $\mathrm{KCl}$ & 25 & 74.55 & 2.86 \\
\hline $\mathrm{CaCl}_{2}{ }^{*} 2 \mathrm{H}_{2} \mathrm{O}$ & 20 & 147.01 & 2.94 \\
\hline $\mathrm{MgSO}_{4}{ }^{*} 7 \mathrm{H}_{2} \mathrm{O}$ & 10 & 246.48 & 1.7 \\
\hline $\mathrm{KH}_{2} \mathrm{PO}_{4}$ & 12.5 & 136.08 & 2.46 \\
\hline
\end{tabular}

Table 1: $10 \mathrm{x}$ slice pre-solution $(1 \mathrm{~L})$. 


\begin{tabular}{|c|c|c|c|}
\hline Compound & Concentration (mM) & Molecular weight (g/mol) & Amount (g) \\
\hline $\mathrm{NaCl}$ & 125 & 58.44 & 7.3 \\
\hline $\mathrm{KCl}$ & 2.5 & from $1 \mathrm{M} \mathrm{CaCl} 2$ solution & 2.19 \\
\hline $\mathrm{CaCl}_{2}{ }^{*} 2 \mathrm{H}_{2} \mathrm{O}$ & 2 & from $1 \mathrm{M} \mathrm{MgSO} 4$ solution & $1 \mathrm{~mL}$ \\
\hline $\mathrm{MgSO}_{4}{ }^{*} 7 \mathrm{H}_{2} \mathrm{O}$ & 1 & 156.02 & 0.2 \\
\hline $\mathrm{NaH}_{2} \mathrm{PO}_{4}{ }^{*} 2 \mathrm{H}_{2} \mathrm{O}$ & 1.25 & 84.01 & 2.18 \\
\hline $\mathrm{NaHCO}$ & 26 & 198.17 & 4.95 \\
\hline Glucose
\end{tabular}

Table 2: 1x ACSF (1 L) (osmolarity between 305-315 mOsm).

\begin{tabular}{|c|c|c|c|}
\hline Compound & Concentration $(\mathbf{m M})$ & Molecular weight (g/mol) & Amount (g) \\
\hline 10x slice presolution & N/A & N/A & $25 \mathrm{~mL}$ \\
\hline Sucrose & 252 & 342.3 & 21.57 \\
\hline NaHCO 3 & 26 & 84.01 & 0.55 \\
\hline Glucose ${ }^{*} \mathrm{H}_{2} \mathrm{O}$ & 10 & 198.17 & 0.49 \\
\hline
\end{tabular}

Table 3: 1x high-sucrose slice solution ( $250 \mathrm{~mL}$ ) (osmolarity between $320-325 \mathrm{mOsm}$ ). 


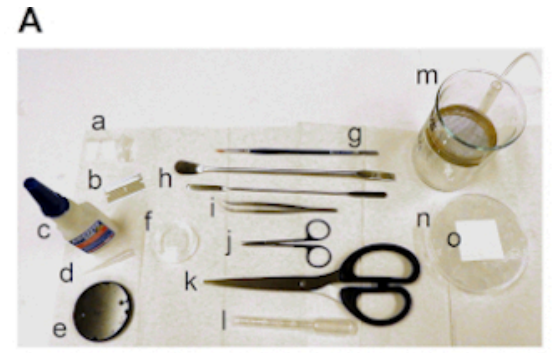

C

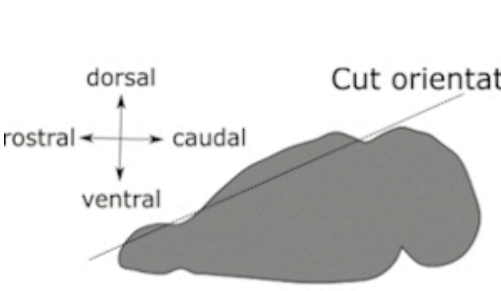

$\mathrm{F}$

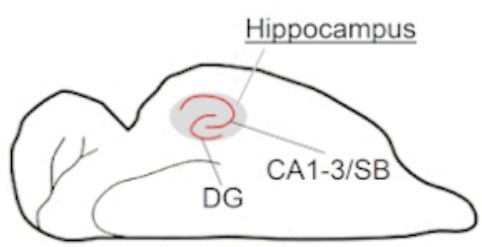

B

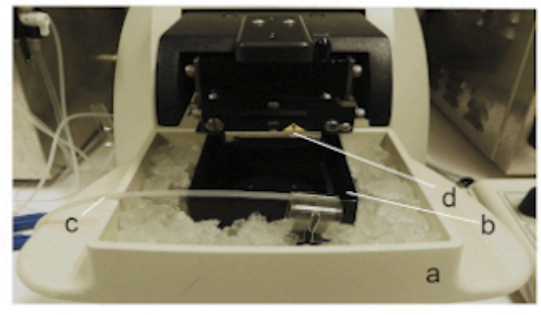

E

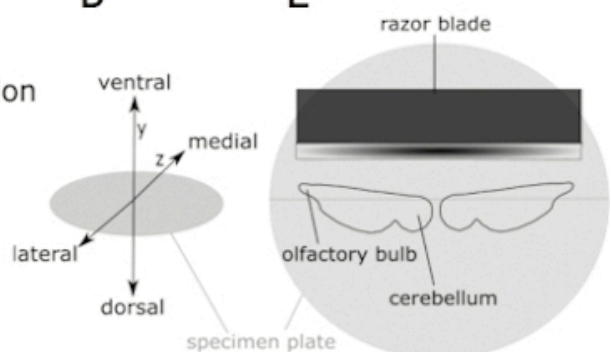

G

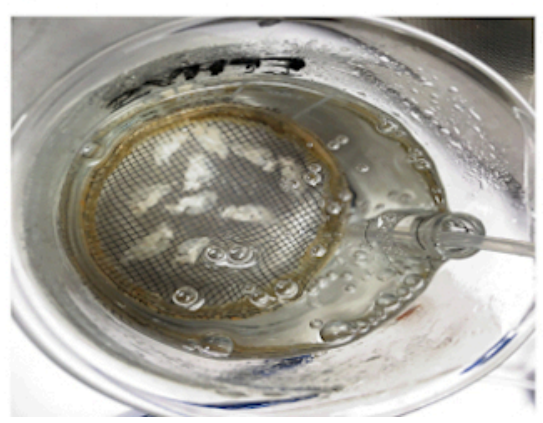

Figure 2: Detailed information on the preparation of horizontal hippocampal brain slices. (A) Image of tools required for dissection and slicing of the rodent brain: (a) $\pm 2 \mathrm{~cm}$ long and $\pm 0.5 \mathrm{~cm}$ wide straps of filter paper (e.g., grade 413); (b) blade; (c) super glue; (d) pipette tip; (e) specimen plate (comes with vibratome); (f) 35 mm culture dish; (g) fine brush; (h) spatula; (i) curved forceps; (j) dissection scissors; (k) strong scissors (blade length above $10 \mathrm{~cm}$ ); (I) plastic Pasteur pipette with wide opening (between 0.6 to $0.8 \mathrm{~cm}$ in diameter); $(\mathrm{m})$ recovery chamber (self-made with $250 \mathrm{~mL}$ beaker, plastic ring, nylon mesh, piece of a $10 \mathrm{~mL}$ serological pipette); (n) $90 \mathrm{~mm}$ culture dish filled with ice and (o) square of filter paper on top of the chilled culture dish. (B) Picture of a vibratome with (a) holder of slice chamber filled with ice; (b) slice chamber; (c) carbogen line and (d) slicing razor blade. (C) Cartoon illustrating the orientation of the cut of the dorsal side of one hemisphere in order to prepare the brain for horizontal slicing (see step 3.9). (D) Isometric projection of the brain orientation on the specimen plate of the vibratome. (E) Cartoon illustrating a top view of the position of the two hemispheres on the specimen plate. (F) Cartoon showing the position of the hippocampus in a horizontal brain slice. The dentate gyrus (DG) and Cornu Ammonis (CA)-Subiculum (SB) regions of the hippocampus are indicated. (G) Picture of a recovery chamber with 
carbogenated ACSF containing ten freshly sliced horizontal brain slices. Please click here to view a larger version of this figure. 
A

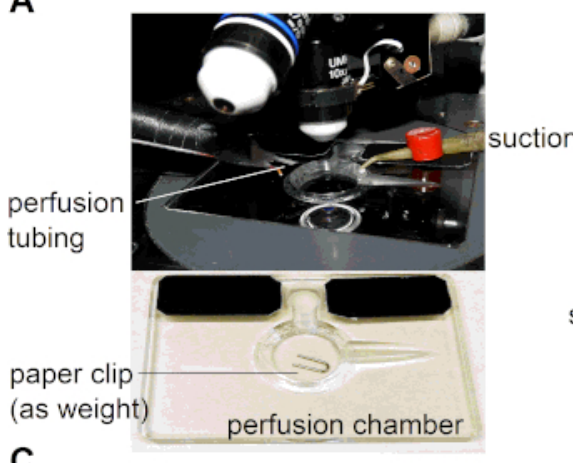

C
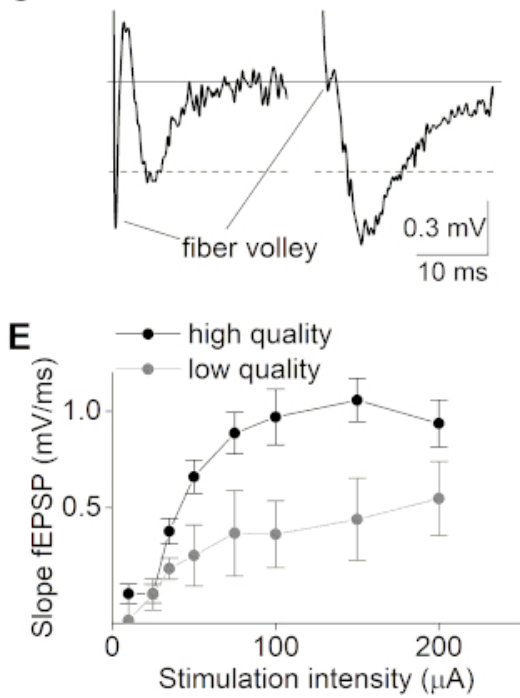

G

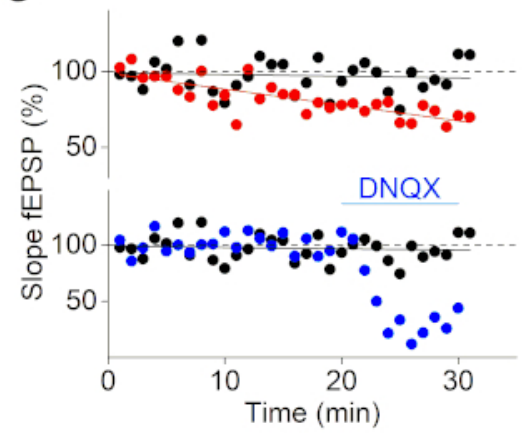

B
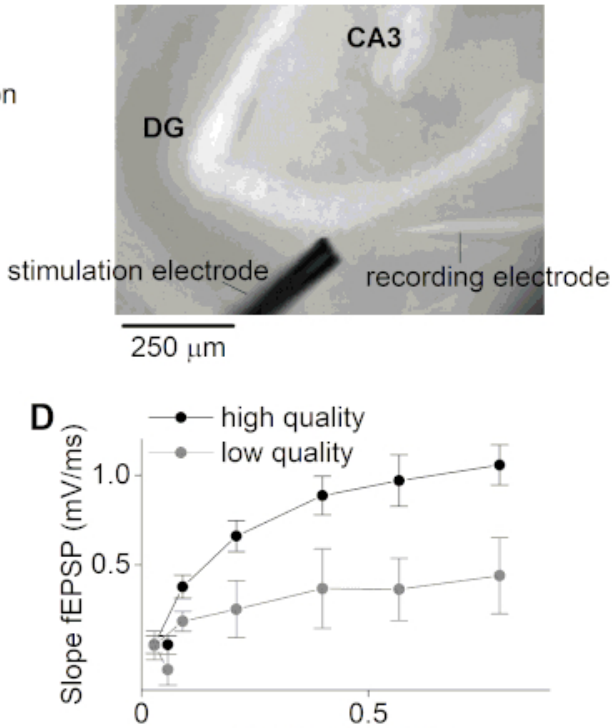

$\mathrm{F}$

Fiber volley $(\mathrm{mV})$

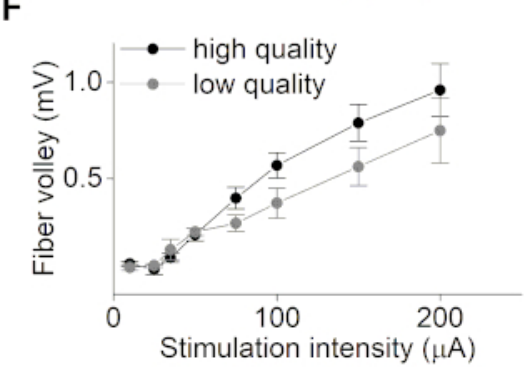

$\mathrm{H}$

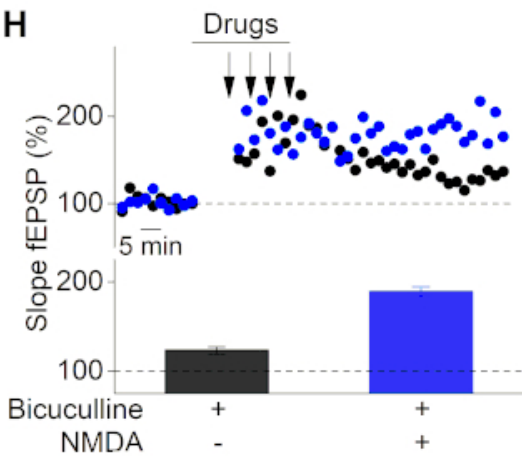

Figure 3: Electrophysiological recordings of hippocampal brain slices. (A) Image of a recording chamber with perfusion and suction, used under an upright microscope. A brain slice will be placed in the chamber and immobilized with a piece of a paper clip before the start of the recordings. (B) Bright field picture of a hippocampal brain slice under an upright microscope (10x objective). The dentate gyrus (DG) and CA3 region are indicated as well as the stimulation (bottom left) and recording (bottom right) electrodes, targeting the medial perforant path during fEPSP recordings. (C) Left: representation of a lowquality slice example of a fEPSP recording with a robust fiber volley and a small amplitude. Right: high quality slice example of a fEPSP recording. The gray line indicates the baseline level. The dotted lines point out the cut-off amplitude of $0.5 \mathrm{mV}$. 
(D) Plot of the fEPSP slope versus the FV amplitude for high quality (black; $n=10$ ) and low-quality brain slices (gray; $n=4)$. Data represented as mean \pm SEM. (E) Input-Output plot (fEPSP slope) for different stimulation intensities $(\mu A)$ for high quality slices (black; $n=10$ ) and low-quality slices (gray; $n=4)$ ). $(F)$ Same as in $(E)$ but for the FV amplitudes versus the stimulus intensities. (G) Time course of three different baseline fEPSP recordings (slope of fEPSP in \%; normalized to the mean fEPSP slope of the first $5 \mathrm{~min}$ ). Upper panel represents a positive (black) and negative (red) example, where the latter has an unstable baseline due to omission of carbogen during the recording. Lower panel shows two stable baseline recordings in treated (after 20 min of stable baseline, AMPA receptors were blocked by application of the AMPA receptor antagonist DNQX $(10 \mu \mathrm{M})$ ) (blue) and untreated condition (black). $(\mathbf{H})$ Time course of LTP recordings for different treatment conditions (indicated in lower panel). Black color for application of Bicuculline (20 $\mu \mathrm{M})$ during conditioning and blue for coapplication of Bicuculline $(20 \mu \mathrm{M})$ and NMDA $(10 \mu \mathrm{M})$ during conditioning. Arrows in upper panel indicate the time points where high frequency stimulation was applied ( $4 \times 1 \mathrm{~s}$ of $100 \mathrm{~Hz}$ ). Bar graph in lower panel represents the mean fEPSP slopes (\%) for 50-60 min after LTP induction of the experiments shown in the upper panel (single representative recording for each condition). Please click here to view a larger version of this figure.

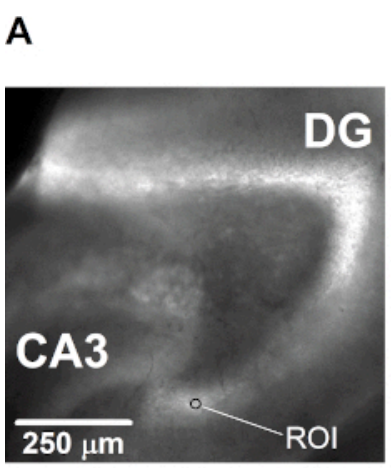

B

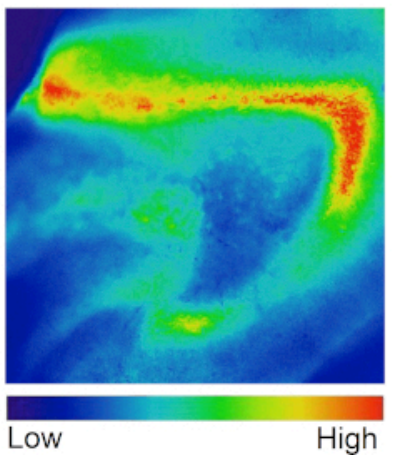

C

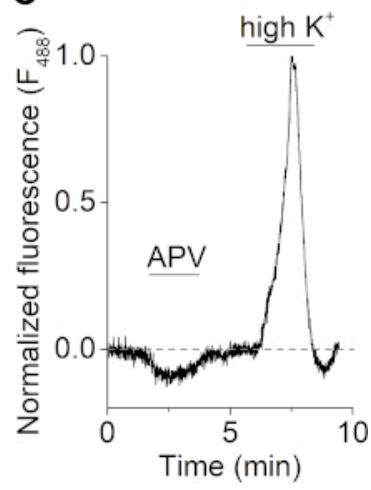

Figure 4: Calcium-microfluorimetry of hippocampal brain slices. (A and B) Fluorescence image (excited at $488 \mathrm{~nm}$ ) (A) and corresponding heat map (B) of a horizontal hippocampal brain slice of the mouse brain. The dentate gyrus (DG), CA3 region, and an example of a region of interest $(\mathrm{ROI})$ are indicated in panel A. (C) Time course of calcium responses $\left(\mathrm{F}_{488} \mathrm{~nm}\right)$ from a ROI in the dentate gyrus of an acute hippocampal brain slice during treatment with the NMDA receptor antagonist APV $(50 \mu \mathrm{M})$ and solution containing high extracellular potassium $\left(\mathrm{K}^{+}\right)(50 \mathrm{mM})$. The trace is normalized to the highest calcium response during high $\mathrm{K}^{+}$perfusion and is baseline corrected for photobleaching. Please click here to view a larger version of this figure. 


\section{Discussion}

Although commonly used among the neuroscience community, brain slice preparations are also faced with several disadvantages. For instance, input and output connections to the brain areas of interest are no longer connected in a brain slice. Moreover, once isolated, the tissue starts degrading slowly over time and this process could alter the physiological conditions of the brain slice. This topic in particular is very concerning because most brain slice recordings are taking several minutes to hours, which results in long experimental days with recordings performed on tissue that was isolated up to $6-8 \mathrm{~h}$ before the start of the experiment. Furthermore, the cerebrospinal fluid and blood circulation get interrupted during slice preparations, which may lead to the lack of important endogenous compounds within a brain slice. And most obviously, the slicing procedure itself may cause mechanical tissue damage that might compromise the obtained results. However, the actual benefits of brain slice preparations are still outweighing their disadvantages, which is why they present a highly valued and employed technique in neuroscience research.

Acute hippocampal brain slices present a powerful and therefore widely used technique to investigate neuronal processes from a molecular level up to complex brain circuit studies. This is based on the ideal neuroanatomy of the hippocampus that can be easily preserved in a slice preparation ${ }^{18}$. Consequently, hippocampal brain slices are used in a wide variety of scientific research projects, including drug screenings ${ }^{17}$, studies of neuronal and synaptic properties involved in cognitive functions 40,41 , and investigations of pathological brain conditions ${ }^{14,42,43}$. However, a broad spectrum of different applications also causes a wide range of available slice preparation protocols that can differ in various parameters, such as dissection conditions and cutting plane orientation, among others. Therefore, the exact research question of a scientific project has to be determined in order to choose an appropriate slice preparation protocol.

The tissue chopper presents one of the oldest used techniques in order to prepare hippocampal brain slices $^{44,45}$. The major advantages of this preparation method include the low cost of the chopper and the fast and easy usage ${ }^{46}$. However, tissue choppers cause mechanical stress that results in morphological alterations and cell death ${ }^{47}$. In comparison, the vibratome is a rather expensive machine and the time for slice preparation is significantly increased which might have an impact on the quality of the slice. However, the vibratome usually offers a more gentle manner of separating the slices from the tissue and allows to keep the brain nicely cooled and oxygenated over the entire isolation procedure, thereby improving slice properties ${ }^{46}$. Therefore, several groups are standardly employing this technique and have brought forward protocols for the preparation of acute hippocampal brain slices using the vibratome $^{16,30,48}$. While some protocols provide only a few details for the slicing itself but rather focus on a specific application of such slice preparation ${ }^{48}$, others provide detailed slice protocols that differ in cutting plane or other protocol details (e.g., agarose embedding or slice/recovery solutions) given in this article 27,30 .

The protocol described here presents a straightforward method in order to prepare high quality acute horizontal hippocampal mouse brain slices from young animals. The protocol is particularly useful to preserve the perforant path (medial and lateral) that presents the hippocampal input pathway, which projects from the entorhinal cortex to the hippocampus $^{8}, 49,50$. Sagittal, coronal, as well as isolated 
hippocampus transverse slice preparations do not properly preserve the perforant path, which originates from mainly Layers II and V of the entorhinal cortex and projects to several areas within the hippocampus ${ }^{18}$. Due to the anatomical positioning of the entorhinal cortex in relation to the hippocampus, horizontal brain slices are a necessity in order to maintain fully intact perforant path fibers within the slice preparation ${ }^{31}$. Additionally, horizontal slicing ideally preserves the mossy fibers that project from the dentate gyrus to the CA3 neurons within the hippocampus $9,30,50$. Therefore, this preparation method is of high value for studies that investigate hippocampal input pathways and DG-related processes. In addition, this protocol allows the investigation of the Schaffer collateral pathway ${ }^{50}$. However, sagittal and coronal brain slice preparations are more commonly used when investigating $\mathrm{CA} 3$ to $\mathrm{CA} 1$ fiber projections, presumably because of their slightly faster preparation time that can increase the chance of obtaining high quality slices. Nevertheless, horizontal hippocampal slice preparations present a powerful research tool since it allows the preservation and investigation of all hippocampal fiber pathways within one slice hemisphere. This can be particularly useful when circuit responses are studied, for example, in multi electrode assay recordings.

A major concern when preparing brain slices is the proper preservation of the brain tissue. This is accomplished by several critical steps in our protocol, including a fast dissection, the continuous and sufficient oxygenation and cooling of the tissue, and the protection of the brain tissue by use of the protective cutting method with a low-sodium, high-sucrose slicing solution 39,51 . Despite the fact that the protocol described here yields a success rate around $90 \%$, potentially additional protective steps might be required when working with tissue derived from older or genetically diverse animals or when trying to preserve a specific cell population. Several methods were already reported to protect sensitive brain tissue preparations. These methods include the use of NMDG-based slicing solutions to reduce the sodium permeation ${ }^{52}$, the use of high magnesium levels in the slicing solution in order to block NMDA receptor activity ${ }^{53}$, and the prolonged use of protective solutions also during the recovery period ${ }^{23}$. All of these measures will result in a reduced excitotoxicity. Additionally, a trans-cardial perfusion with ice-cold protective ACSF solutions is often employed and necessary when working with older animals ${ }^{27}$.

Acute hippocampal brain slices are ideally suited and extensively used for electrophysiological studies for reasons such as the high amplitude signals that can be obtained from a relatively thick $(300-500 \mu \mathrm{m})$ acute brain slice, which guarantees a high signal to noise ratio ${ }^{11}$. Standardly used electrophysiological applications include extracellular field recordings and intracellular whole-cell recordings in a voltage- or current-clamp mode. In order to acquire high quality electrophysiological data, the slice health is of primary concern and can be guaranteed by strictly following the presented protocol. However, as slice preparations present a highly sensitive technique, a quality check should be routinely included before the start of each experiment. Several parameters can be used as quality check of the slice and are standardly assessed via Input-Output curves and baseline fEPSP or EPSC recordings ${ }^{19}$. Nevertheless, it should be noted that suboptimal electrophysiological properties can arise from experimental errors such as electrode positioning, orientation or even damage and do not solely represent the health of the prepared slice. Therefore, it is advisable to perform additional quality controls such as simple visualization and assessment of the cells under a $40 \mathrm{x}$ objective or a DAPI nucleus staining. Such quality checks can 
be used to confirm constant slice health over several slice preparation sessions.

Calcium microfluorimetry presents a less commonly used technique to study hippocampal brain slices. However, this technique is of additional value to the standard extracellular and intracellular electrode recordings, as it allows to visualize and quantify intracellular calcium fluxes, which are of high importance in neuronal and synaptic signaling. Changes in intracellular calcium concentrations are involved in neurotransmitter vesicle release, postsynaptic potential generation, regulation of synaptic plasticity and axonal nerve conduction $^{54,55,56}$. As an illustration of this technique (Figure 4), we made use of a commercially available calcium dye. Inarguably, treatment of tissue slices with calcium dyes can yield difficulties such as an increased experimental time frame as well as inefficient loading of lower situated neuronal cells. However, variations on this technique could be used to circumvent these technical challenges. For instance, it is possible to combine calcium measurements and patch clamp recordings in hippocampal slices. In this way, a calcium fluorescent dye could be loaded into a specific cell through the patch pipette, allowing the measurements of calcium dynamics in one specific cell of interest ${ }^{57}$. Alternatively, genetically engineered animals expressing the calcium indicator, GCaMP ${ }^{58}$, either in the whole brain, or driven by a cell-specific promotor, could be used. Interestingly, brain tissue from GCaMP animals with a direct linker to a protein of interest could provide opportunities to determine the neuronal expression pattern or investigate the involvement in calcium sparks and waves.

Altogether, we provide the guidelines for the successful preparation of healthy and viable horizontal hippocampal brain slices from mice for electrophysiological and imaging recordings. This methodology is very useful to access neurological changes that occur in brain pathologies that are described in the dentate gyrus.

\section{Disclosures}

The authors have nothing to disclose.

\section{Acknowledgments}

We thank the Electrophysiology unit of the VIB-KU Leuven Center for Brain and Disease Research under the supervision of Dr. Keimpe Wierda and Prof. Dr. Joris De Wit for the use of their research facilities. Furthermore, we thank all the members of the Laboratory of Ion Channel Research and the Laboratory of Endometrium, Endometriosis and Reproductive Medicine at the KU Leuven for their helpful discussions and comments.

This project has received funding from the Research Foundation-Flanders (G.084515N and G.0B1819N to J.V.) and the Research Council of the KU Leuven (C1-funding C14/18/106 to J.V.). K.P. is a FWO [PEGASUS] ${ }^{2}$ Marie Skłodowska-Curie Fellow and received funding from the European Union's Horizon 2020 research and innovation program under the Marie Skłodowska-Curie grant agreement (665501) with the Research Foundation Flanders (FWO) (12T0317N). K.H. is a Postdoctoral Fellow of the Research Foundation Flanders, Belgium (12U7918N).

\section{References}

1. Scoville, W. B., Milner, B. Loss of recent memory after bilateral hippocampal lesions. Journal of Neurology, Neurosurgery, and Psychiatry. 20 (1), 11-21 (1957).

2. Cavarsan, C. F., Malheiros, J., Hamani, C., Najm, I., Covolan, L. Is mossy fiber sprouting a potential 
therapeutic target for epilepsy? Frontiers in Neurology. $\mathbf{9}$ 1023 (2018).

3. Nadler, J. V. The recurrent mossy fiber pathway of the epileptic brain. Neurochemical Research. 28 (11), 1649-1658 (2003).

4. Raimondo, J. V. et al. Methodological standards for in vitro models of epilepsy and epileptic seizures. A TASK1WG4 report of the AES/ILAE translational task force of the ILAE. Epilepsia. 58 (Suppl 4), 40-52 (2017).

5. Humpel, C. Organotypic brain slice cultures: A review. Neuroscience. 305, 86-98 (2015).

6. Tohno, Y. et al. Relationships among the hippocampus, dentate gyrus, mammillary body, fornix, and anterior commissure from a viewpoint of elements. Biological Trace Element Research. 140 (1), 35-52 (2011).

7. Maclean, P. D. The limbic system and its hippocampal formation; studies in animals and their possible application to man. Journal of Neurosurgery. 11 (1), 29-44 (1954).

8. Petersen, R. P. et al. Electrophysiological identification of medial and lateral perforant path inputs to the dentate gyrus. Neuroscience. 252, 154-168 (2013).

9. Amaral, D. G., Scharfman, H. E., Lavenex, P. The dentate gyrus: fundamental neuroanatomical organization (dentate gyrus for dummies). Progress in Brain Research. 163, 3-22 (2007).

10. Szirmai, I., Buzsaki, G., Kamondi, A. 120 years of hippocampal schaffer collaterals. Hippocampus. 22 (7), 1508-1516 (2012).

11. Cho, S., Wood, A., Bowlby, M. R. Brain slices as models for neurodegenerative disease and screening platforms to identify novel therapeutics. Current Neuropharmacology. 5 (1), 19-33 (2007).

12. Mathis, D. M., Furman, J. L., Norris, C. M. Preparation of acute hippocampal slices from rats and transgenic mice for the study of synaptic alterations during aging and amyloid pathology. Journal of Visualized Experiments. (49) e2330 (2011).

13. Papouin, T., Haydon, P. G. Obtaining Acute Brain Slices. Bio-protocol. 8 (2), e2699 (2018).

14. Li, Q., Han, X., Wang, J. Organotypic hippocampal slices as models for stroke and traumatic brain injury. Molecular Neurobiology. 53 (6), 4226-4237 (2016).

15. Lo, D. C., McAllister, A. K., Katz, L. C. Neuronal transfection in brain slices using particle-mediated gene transfer. Neuron. 13 (6), 1263-1268 (1994).

16. Lein, P. J., Barnhart, C. D., Pessah, I. N. Acute hippocampal slice preparation and hippocampal slice cultures. Methods in Molecular Biology (Clifton, N.J.). 758 115-134 (2011).

17. Magalhaes, D. M. et al. Ex vivo model of epilepsy in organotypic slices-a new tool for drug screening. Journal of Neuroinflammation. 15 (1), 203 (2018).

18. Bliss, T. in The hippocampus book. eds Per Andersen, Richard Morris, David Amaral, John O'Keefe. Ch. 3, 37114, Oxford University Press. (2007).

19. Bortolotto, Z. A., Amici, M., Anderson, W. W., Isaac, J. T. R., Collingridge, G. L. Synaptic plasticity in the hippocampal slice preparation. Current Protocols in Neuroscience. 54 (1), 6.13.11-16.13.26 (2011).

20. Al-Osta, I. et al. Imaging calcium in hippocampal presynaptic terminals with a ratiometric calcium sensor 
in a novel transgenic mouse. Frontiers in Cellular Neuroscience. 12, 209 (2018).

21. McLeod, F., Marzo, A., Podpolny, M., Galli, S., Salinas, P. Evaluation of synapse density in hippocampal rodent brain slices. Journal of Visualized Experiments. (128) e56153 (2017)

22. Segev, A., Garcia-Oscos, F., Kourrich, S. Wholecell patch-clamp recordings in brain slices. Journal of Visualized Experiments. (112), e54024 (2016).

23. Ting, J. T., Daigle, T. L., Chen, Q., Feng, G. Acute brain slice methods for adult and aging animals: application of targeted patch clamp analysis and optogenetics. Methods in Molecular Biology (Clifton, N.J.). 1183, 221-242 (2014).

24. Weng, W., Li, D., Peng, C., Behnisch, T. Recording synaptic plasticity in acute hippocampal slices maintained in a small-volume recycling-, perfusion-, and submersiontype chamber system. Journal of Visualized Experiments. (131), e55936 (2018).

25. Zhou, Q., Abe, H., Nowak, T. S., Jr. Immunocytochemical and in situ hybridization approaches to the optimization of brain slice preparations. Journal of Neuroscience Methods. 59 (1), 85-92 (1995).

26. Koike-Tani, M., Tominaga, T., Oldenbourg, R., Tani, T. Birefringence changes of dendrites in mouse hippocampal slices revealed with polarizing microscopy. Biophysical Journal. 110 (10) 2366-2384 (2020).

27. Ting, J. T. et al. Preparation of acute brain slices using an optimized N-Methyl-D-glucamine protective recovery method. Journal of Visualized Experiments. (132), e53825 (2018).
28. Zhao, S. et al. Cell type-specific channelrhodopsin-2 transgenic mice for optogenetic dissection of neural circuitry function. Nature Methods. 8 (9), 745-752 (2011).

29. Hua, Y., Keep, R. F., Hoff, J. T., Xi, G. Brain injury after intracerebral hemorrhage: the role of thrombin and iron. Stroke. 38 (2 Suppl), 759-762 (2007).

30. Bischofberger, J., Engel, D., Li, L., Geiger, J. R., Jonas, P. Patch-clamp recording from mossy fiber terminals in hippocampal slices. Nature Protocols. 1 (4), 2075-2081 (2006).

31. Paxinos, G., Franklin, K. The Mouse Brain In Stereotaxic Coordinates. 3 edn, 256. Academic Press (2008).

32. Lacar, B., Young, S. Z., Platel, J. C., Bordey, A. Preparation of acute subventricular zone slices for calcium imaging. Journal of Visualized Experiments. (67), e4071 (2012)

33. Schauer, C., Leinders-Zufall, T. Imaging calcium responses in GFP-tagged neurons of hypothalamic mouse brain slices. Journal of Visualized Experiments. (66), e4213 (2012).

34. Tetteh, H., Lee, J., Lee, J., Kim, J. G., Yang, S. Investigating Long-term Synaptic Plasticity in Interlamellar Hippocampus CA1 by Electrophysiological Field Recording. Journal of Visualized Experiments. (150), e59879 (2019).

35. Smith, C. J. et al. Investigations on alterations of hippocampal circuit function following mild traumatic brain injury. Journal of Visualized Experiments. (69), e4411 (2012)

36. McNaughton, B. L. Evidence for two physiologically distinct perforant pathways to the fascia dentata. Brain Research. 199 (1), 1-19 (1980). 
37. Colino, A., Malenka, R. C. Mechanisms underlying induction of long-term potentiation in rat medial and lateral perforant paths in vitro. Journal of Neurophysiology. 69 (4), 1150-1159 (1993).

38. Coulter, D. A., Carlson, G. C. Functional regulation of the dentate gyrus by GABA-mediated inhibition. Progress in Brain Research. 163, 235-243 (2007).

39. Kuenzi, F. M., Fitzjohn, S. M., Morton, R. A., Collingridge, G. L., Seabrook, G. R. Reduced long-term potentiation in hippocampal slices prepared using sucrose-based artificial cerebrospinal fluid. Journal of Neuroscience Methods. 100 (1-2), 117-122 (2000).

40. Connor, S. A. et al. Loss of synapse repressor MDGA1 enhances perisomatic inhibition, confers resistance to network excitation, and impairs cognitive function. Cell Reports. 21 (13), 3637-3645 (2017).

41. Lisman, J. et al. Viewpoints: how the hippocampus contributes to memory, navigation and cognition. Nature Neuroscience. 20 (11), 1434-1447 (2017).

42. Moodley, K. K., Chan, D. The hippocampus in neurodegenerative disease. Frontiers of Neurology and Neuroscience. 34, 95-108 (2014).

43. Kong, $H$. et al. Inhibition of miR-181a-5p reduces astrocyte and microglia activation and oxidative stress by activating SIRT1 in immature rats with epilepsy. Laboratory Investigation; A Journal of Technical Methods and Pathology. (2020).

44. Skrede, K. K., Westgaard, R. H. The transverse hippocampal slice: a well-defined cortical structure maintained in vitro. Brain Research. 35 (2), 589-593 (1971).
45. Schwartzkroin, P. A. Characteristics of CA1 neurons recorded intracellularly in the hippocampal in vitro slice preparation. Brain Research. 85 (3), 423-436 (1975).

46. Wang, T., Kass, I. S. Preparation of brain slices. Methods in Molecular Biology (Clifton, N.J.). 72, 1-14 (1997).

47. Garthwaite, J., Woodhams, P. L., Collins, M. J., Balazs, R. On the preparation of brain slices: morphology and cyclic nucleotides. Brain Research. 173 (2), 373-377 (1979).

48. Booker, S. A., Song, J., Vida, I. Whole-cell patch-clamp recordings from morphologically- and neurochemically-identified hippocampal interneurons. Journal of Visualized Experiments: JoVE. (91), e51706 (2014).

49. Aydin-Abidin, S., Abidin, I. 7,8-Dihydroxyflavone potentiates ongoing epileptiform activity in mice brain slices. Neuroscience Letters. 703, 25-31 (2019).

50. Xiong, G., Metheny, H., Johnson, B. N., Cohen, A. S. A comparison of different slicing planes in preservation of major hippocampal pathway fibers in the mouse. Frontiers in Neuroanatomy. 11, 107 (2017).

51. Aghajanian, G. K., Rasmussen, K. Intracellular studies in the facial nucleus illustrating a simple new method for obtaining viable motoneurons in adult rat brain slices. Synapse. 3 (4), 331-338 (1989).

52. Tanaka, Y., Tanaka, Y., Furuta, T., Yanagawa, Y., Kaneko, T. The effects of cutting solutions on the viability of GABAergic interneurons in cerebral cortical slices of adult mice. Journal of Neuroscience Methods. 171 (1), 118-125 (2008).

53. Reid, K. H., Edmonds, H. L., Jr., Schurr, A., Tseng, M. T., West, C. A. Pitfalls in the use of brain slices. Progress in Neurobiology. 31 (1), 1-18 (1988). 
54. Brini, M., Calì, T., Ottolini, D., Carafoli, E. Neuronal calcium signaling: function and dysfunction. Cellular and Molecular Life Sciences: CMLS. 71 (15), 2787-2814 (2014).

55. Gleichmann, M., Mattson, M. P. Neuronal calcium homeostasis and dysregulation. Antioxidants \& Redox Signaling. 14 (7), 1261-1273 (2011).

56. Padamsey, Z., Foster, W. J., Emptage, N. J. Intracellular $\mathrm{Ca}(2+)$ release and synaptic plasticity: a tale of many stores. The Neuroscientist: A Review Journal Bringing Neurobiology, Neurology and Psychiatry. 25 (3), 208-226 (2019).

57. Chen-Engerer, H.J. et al. Two types of functionally distinct $\mathrm{Ca} 2+$ stores in hippocampal neurons. Nature Communications. 10 (1), 3223 (2019).

58. Akerboom, J. et al. Optimization of a GCaMP calcium indicator for neural activity imaging. The Journal of Neuroscience: The Official Journal of the Society for Neuroscience. 32 (40), 13819-13840 (2012). 